\title{
ORIGINAL
}

\section{ENFERMEDAD INVASORA POR HAEMOPHILUS INFLUENZAE ANTES Y DESPUÉS DE LA CAMPAÑA DE VACUNACIÓN EN LA POBLACIÓN INFANTIL DE LA COMUNIDAD VALENCIANA (1996-2000)}

Mercedes Goicoechea Sáez (1), Ana M. ${ }^{a}$ Fullana Montoro (1), Pilar Momparler Carrasco (1), M. ${ }^{a}$ Jesús Redondo Gallego (1), Juan Brines Solanes (2) y Francisco J. Bueno Cañigral (3).

(1) Servicio de Salud Infantil y de la Mujer. Dirección General de Salud Pública. Conselleria de Sanidad. Comunidad Valenciana.

(2) Servicio de Pediatría. Hospital Clínico Universitario de Valencia.

(3) Concejalía de Sanidad y Consumo. Ayuntamiento de Valencia.

\section{RESUMEN}

Fundamento: La introducción de la vacuna conjugada anti Haemophilus influenzae tipo b (Hib) en niños ha provocado un llamativo descenso de la incidencia de la enfermedad por $H$. influenzae. El objetivo de este estudio es analizar las características más relevantes de la enfermedad invasora por $H$. influenzae en cuanto a la epidemiología, clínica, evolución y estado de vacunación de la población infantil de la Comunidad Valenciana en el periodo 1996-2000.

Método: Los datos se recogen de las historias clínicas de los niños menores de 15 años que hayan presentado síntomas y signos clínicos sugestivos de enfermedad invasora con aislamiento de Haemophilus influenzae y/o que cumple con los criterios de definición de caso establecidos, atendidos en todos los hospitales públicos de la Comunidad Valenciana entre 1996 y 2000. La evolución de la incidencia se valoró mediante tasas de incidencia. La clínica y su evolución (secuelas y letalidad) mediante la frecuencia y distribución por edad.

Resultados: Se registraron un total de 36 casos de enfermedad invasora por Haemophilus influenzae. La tasa de incidencia en niños menores de 15 años pasó de $3,56 / 10^{5}$ en 1996 a $1,07 / 10^{5}$ en 1997 (coincidiendo con la campaña de vacunación y la posterior inclusión de la vacuna conjugada anti Hib en el Calendario de Vacunaciones Sistemáticas de la Comunidad Valenciana) y $0,30 / 10^{5}$ en 1998 , situación que se sigue manteniendo en los años posteriores. El 53\% de los casos se dan en menores de 18 meses. Tanto las secuelas como los fallecimientos se producen en la época anterior a la aplicación rutinaria de la vacuna conjugada. Ningún niño vacunado correctamente falleció. Se registraron 2 casos de $H$. influenzae tipo no b en niños vacunados.

Conclusiones: La incidencia de la infección por Haemophilus influenzae tipo b disminuyó drásticamente desde el inicio de la vacunación sistemática de la población infantil.

Palabras clave: Haemophilus influenzae tipo b. Infancia. Vacunas. Incidencia. Mortalidad.

Correspondencia:

Mercedes Goicoechea Sáez

P1. Poeta Eduardo Marquina n. ${ }^{\circ} 4$ pta $20 .^{\text {a }}$

46014 Valencia

Correo electrónico: mergoico@terra.es

\section{ABSTRACT}

Invasive Disease due to Haemophilus Influenzae before and after the Immunization Campaign among the Infantile Population in the Autonomous Community of Valencia (1996-2000)

Background: The introduction of a conjugate type b Haemophilus influenzae (Hib) vaccine for children has led to a sharp drop in the incidence of $H$. influenzae disease. The purpose of this study is that of analyzing the major characteristics of invasive disease due to $H$. influenzae as regards epidemiology, clinical aspects, evolution and immunization status among the infantile population of the Autonomous Community of Valencia for the 1996-2000 period.

Method: The data was taken from the clinical records of those children under age 15 , who have shown clinical signs and symptoms indicative of invasive disease entailing isolation of Haemophilus influenzae and/or meeting the established case definition requirements, who were treated at all of the public hospitals in the Autonomous Community of Valencia throughout the 1996-2000 period. The trend of incidence was assessed by mean of incidence rates. The clinical pattern and the evolution thereof (sequelae and life-threatening aspects) by frequency and age range.

Results: A total of 36 cases of invasive disease due to Haemophilus influenzae were recorded. The incidence rate among children under age 15 dropped from $3.56 / 10^{5}$ in 1996 to $1.07 / 10^{5}$ in 1997 (coinciding with the immunization campaign and the subsequent including of the conjugate Hib vaccine in the Routine Vaccination Schedule of the Autonomous Community of Valencia) and $0.30 / 10^{5}$ in 1998 , this being a situation which has continued over the following years. Fifty-three percent $(53 \%)$ of the cases occur in children under age 18 months. Both the sequelae as well as the deaths occurred throughout the period prior to the routine use of the conjugate vaccine. No child properly immunized died. Two cases of non-b type $H$. influenzae occurred in immunized children.

Conclusions: The incidence of infection due to type b Haemophilus influenzae dropped drastically as of the start of the routine immunization of the infantile population.

Keywords: Type b Haemophilus influenzae type b. Infant. Vaccines. Incidence. Mortality. 


\section{INTRODUCCIÓN}

Haemophilus influenzae es una bacteria que coloniza habitualmente la rinofaringe de los niños y jóvenes de forma asintomática. La capacidad de invasión y multiplicación está estrechamente relacionada con el estado inmunitario del huésped y con el polisacárido capsular del germen que es el responsable de su virulencia, siendo el serotipo b el responsable del $90-95 \%$ de la enfermedad invasora ${ }^{1}$.

La infección ocasiona típicamente un proceso infeccioso de carácter agudo. La meningitis aguda purulenta es la presentación más frecuente, siendo el cuadro clínico en los primeros momentos indistinguible del provocado por otros microorganismos causales como $N$. meningitidis o $S$. pneumoniae. Otros cuadros clínicos de enfermedad invasora son la sepsis, la neumonía, infección osteoarticular, peritonitis, epiglotitis y celulitis $^{2-4}$.

La incidencia de la enfermedad invasora bacteriana por Haemophilus influenzae varía según la edad, siendo los más jóvenes los más afectados, especialmente los menores de 2 años. También la incidencia es mayor cuando existen factores predisponentes, es decir que la infección puede tener carácter oportunista ${ }^{4,5}$.

H. influenzae tipo b (Hib) tiene la capacidad de generar una respuesta inmunológica en los individuos a partir de sus antígenos capsulares, lo que ha posibilitado el desarrollo de vacunas. Las vacunas de tipo conjugado, en las que los antígenos capsulares se unen a una proteína transportadora, subsanan las limitaciones de las vacunas polisacarídicas ${ }^{6}$. Al contrario que éstas últimas, las vacunas conjugadas tienen la capacidad de inducir respuesta inmune a partir de los primeros meses de vida y de generar una memoria inmunológica en los vacunados. Igualmente, producen respuesta anamnésica ante dosis de refuerzo y ofrecen una protección prolongada ${ }^{7-9}$. Estos factores, junto con la observación de que la inmunogenicidad y seguridad de la vacuna son similares cuando se administra sola o con otras vacunas ${ }^{10,11}$, como se indica en las fichas técnicas de las vacunas que contienen Haemophilus y que actualmente están comercializadas en España, permiten su inclusión en el calendario de vacunaciones sistemáticas infantiles, mediante la siguiente pauta vacunal: primovacunación (2, 4, 6 meses) y dosis de refuerzo (a los 18 meses) ${ }^{12}$, siguiendo recomendaciones suficientemente contrastadas en la bibliografía internacional ${ }^{13,14}$. Consecuentemente, la incidencia de esta enfermedad invasora ha disminuido espectacularmente en aquellos países en los que se ha aplicado masivamente integrándola en el calendario vacunal ${ }^{15,16}$.

En la Comunidad Valenciana no existen datos fidedignos de la incidencia real de la enfermedad invasora por $H$. influenzae tipo b, al no haber sido una enfermedad de declaración obligatoria (EDO) hasta 1997, año en que se adoptan las definiciones de caso de todas las enfermedades sometidas a vigilancia, aunque anteriormente a esta fecha se basaban en el criterio de sospecha clínica ${ }^{17}$. En la década de los 90, en diversos estudios realizados en la Comunidad Valenciana ${ }^{18}$, se aprecia una considerable incidencia de enfermedad invasora por $H$. influenzae tipo b, llegando a alcanzar durante el periodo 1994-1995 una tasa de 13,68/100.000 habitantes menores de 5 años de edad, cifra similar o algo inferior a la encontrada en otras Comunidades Autónomas en este mismo periodo $^{4,} 19-22$.

El análisis de la situación clínico-epidemiológica y la disponibilidad de una vacuna conjugada eficaz desde los primeros meses de vida para el $H$. influenzae b, hizo que la Conselleria de Sanitat de la Generalitat Valenciana ofertara (entre el 29 de septiembre y el 11 de diciembre de 1997) la vacunación gratuita frente a $H$. influenzae b a todos los niños/as menores de 5 años, para lo que se empleó la vacuna conjugada con el toxoide tetánico PRP-T, seguida de la inclusión de 
ésta en el Calendario de Vacunaciones Sistemáticas de la Comunidad Valenciana a partir de diciembre de $1997^{23}$. En España, la inclusión de esta vacuna en el calendario de vacunaciones infantiles fue una decisión adoptada por el pleno del Consejo Interterritorial del Sistema Nacional de Salud celebrado el 15 de diciembre de 1997. Esta inclusión se hizo efectiva a lo largo de 1998.

Los objetivos del presente estudio clínico y epidemiológico han sido: 1) Conocer las características más notables en cuanto a la incidencia, distribución etaria, presencia de factores predisponentes o antecedentes de problemas de salud, presentación clínica $y$ evolución de la enfermedad bacteriana invasora por Haemophilus influenzae en la población de 0 a 14 años de edad de la Comunidad Valenciana durante el periodo de 1996 a 2000. 2) Valorar la efectividad de las medidas de vacunación adoptadas en la prevención de la enfermedad invasora por $H$. influenzae en la Comunidad Valenciana, iniciadas a partir del último trimestre de 1997.

\section{SUJETOS Y MÉTODOS}

Se ha diseñado un estudio descriptivo de todos los casos de enfermedad bacteriana invasora por Haemophilus influenzae en niños/as de 1 mes a 14 años de edad inclusive, atendidos entre el 1 de enero de 1996 y el 31 de diciembre de 2000 en los Servicios de Pediatría de los 18 hospitales públicos (con asistencia pediátrica) de la Comunidad Valenciana.

La información se ha obtenido de las historias clínicas (localizadas a través de los registros internos de los Servicios de Pediatría) de los niños que cumplían uno o más de los siguientes criterios de definición de caso:

1. Cuadro clínico infeccioso compatible con enfermedad bacteriana invasora (meningitis, septicemia, neumonía, pleuritis, celulitis, artritis, osteomielitis, bacteriemia, epiglotitis y peritonitis) y aislamiento de Haemophilus in- fluenzae, en un lugar normalmente estéril (sangre, LCR, líquido pleural, derrame articular, aspirado de tejido celular subcutáneo, etc.).

2. Presencia de bacilos gram negativos polimórficos en líquido cefalorraquídeo (LCR) y/o detección de antígeno $H$. influenzae tipo b en LCR, sangre, orina en un paciente con signos clínico-analíticos característicos de enfermedad invasora y cultivos negativos para cualquier otro germen.

La información se recogió a través de la cumplimentación de un cuestionario en formato impreso y en formato informático (ACCES), diseñado para este estudio. Se recogieron en cada uno de los casos datos de filiación, edad, sexo, duración de la estancia en UCI y estancia total en el hospital, manifestaciones clínicas y datos analíticos al ingreso, otras manifestaciones de enfermedad invasora a lo largo de la evolución, tratamiento, aparición de complicaciones y secuelas, fallecimiento, antecedentes de problemas de salud, vacunación frente a Haemophilus influenzae (dato que se recoge de la historia clínica y/o de la cartilla de salud infantil del niño) y antibioterapia previa al ingreso.

Una vez cumplimentado el formulario, los datos se introdujeron en la base de datos (ACCES), procediéndose previamente a la codificación de algunas variables. Periódicamente se realizó un análisis de inconsistencias internas de los datos realizando, en su caso, las correcciones oportunas. Los datos se procesaron en una hoja de cálculo informatizada (EXCEL), para su posterior análisis estadístico y representación gráfica.

Se determinaron: 1) Las tasas de incidencia de la enfermedad por año, las cuales se calcularon a partir de los resultados obtenidos en la población menor de 15 años según el Padrón de 1996 (652.269 personas). 2) La frecuencia y distribución de la infección por edad, sexo, antecedentes de problemas de salud, presentación clínica (número de ca- 
sos y porcentaje) y la evolución (secuelas y letalidad). 3) La estancia hospitalaria y en Unidad de Cuidados Intensivos (media y desviación típica). 4) La efectividad vacunal de los años 1998, 1999 y 2000 mediante la fórmula de Orestein ${ }^{24}$ :

$\mathrm{EV}=[($ Tasa en $\mathrm{NO}$ vacunados - Tasa en vacunados)/Tasa en NO vacunados] $\times 100$.

\section{RESULTADOS}

Durante los 5 años del estudio se registró un total de 36 casos de enfermedad invasora por $H$. influenzae, lo que representa una tasa anual promedio de 1,10 por 100.000 habitantes menores de 15 años de edad para el conjunto del periodo estudiado $(3,56 ; 1,07$; 0,$30 ; 0,15$ y 0,30 para $1996,1997,1998$,
1999 y 2000 respectivamente). Se refleja un drástico descenso de la incidencia a partir de 1997 coincidiendo con la amplia vacunación de la población. La infección presenta un patrón estacional con máxima incidencia en invierno y primavera (figura 1).

La distribución por sexo fue de 20 casos en niños (55\%) y 16 en niñas (45\%), siendo la razón por sexos de 1,3. La distribución por edad queda reflejada en la tabla 1 . Como se aprecia en ella el mayor número de casos se da antes de los 5 años de edad (94\% del total de casos) y especialmente antes de los 18 meses ( $53 \%$ del total de casos). El número de casos disminuye a medida que aumenta la edad.

La presencia de condición médica predisponente $\mathrm{y} / \mathrm{o}$ antecedentes de problemas de

Figura 1

Casos de enfermedad invasora por H. influenzae durante los años 1996-2000 en la Comunidad Valenciana. Distribución por meses

$\mathrm{N}^{\circ}$ CASOS

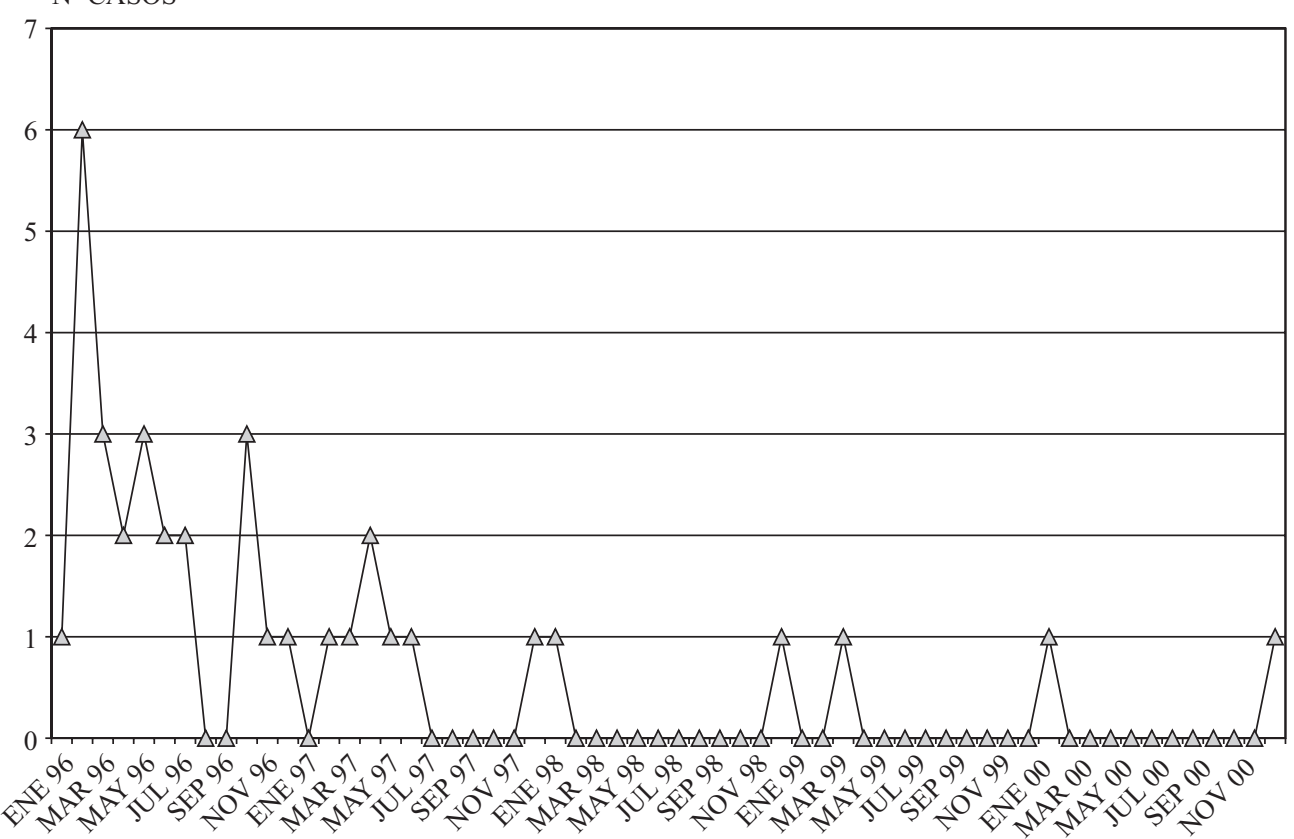


Tabla 1

Distribución de la enfermedad invasora por $H$. influenzae por edad y año de presentación (número de casos y porcentaje)

\begin{tabular}{|c|c|c|c|c|c|c|}
\hline Edad & 1996 & 1997 & 1998 & 1999 & 2000 & Total \\
\hline $1 \mathrm{~m}-18$ meses & $15(62,5 \%)$ & $2(28,6 \%)$ & $1(50,0 \%)$ & & $1(50,0 \%)$ & $19(52,8 \%)$ \\
\hline $19 \mathrm{~m}-4$ años & $8(33,3 \%)$ & $5(71,4 \%)$ & $1(50,0 \%)$ & & $1(50,0 \%)$ & $15(41,6 \%)$ \\
\hline 5 a-9 años & $1(4,2 \%)$ & & & & & $1(2,8 \%)$ \\
\hline $10 \mathrm{a}-14$ años & & & & $1(100 \%)$ & & $1(2,8 \%)$ \\
\hline Total & $24(100 \%)$ & $7(100 \%)$ & $2(100 \%)$ & $1(100 \%)$ & $2(100 \%)$ & $36(100 \%)$ \\
\hline
\end{tabular}

salud queda recogida en la tabla 2 . Se documentó un total de 4 casos con enfermedad

Tabla 2

Antecedentes de problema de salud en los niños/as con enfermedad invasora por $H$. influenzae

\begin{tabular}{|lccc|}
\hline & 1996 & 1997 & Total \\
\hline $\begin{array}{l}\text { Inmunodeficiencia } \\
\text { primaria }\end{array}$ & 1 & & \\
$\begin{array}{l}\text { Malnutrición } \\
\text { Cardiopatía congénita }\end{array}$ & 1 & & $\mathbf{1}$ \\
Otras * & 2 & 1 & $\mathbf{1}$ \\
\hline Total & $\mathbf{4}$ & $\mathbf{2}$ & $\mathbf{3}$ \\
\hline & (en $\mathbf{3}$ niños) & (en 1 niño) & $\mathbf{6}$ \\
\hline
\end{tabular}

* Corresponden a diabetes mellitus (1), Sdr de Dandy-Walker (1), válvula de derivación ventrículo peritoneal por meningitis neonatal (1).

de base en el total del periodo de estudio, correspondientes todos ellos a los años 1996 y 1997 , lo que representa un $11,1 \%$ de los niños con enfermedad invasora por Hib. Tres de ellos tenían menos de 2 años de edad y el otro 3 años y 4 meses.

La presentación clínica de la enfermedad invasora por $H$. influenzae en la infancia se refleja en la tabla 3 . La meningitis fue la presentación clínica más frecuente en el total de los 5 años del estudio (67\%), seguida de la sepsis/bacteriemia (25\%) y de la neumonía (8\%). En el $28 \%$ de los casos se presentó sólo con cuadro clínico de meningitis y en el $39 \%$ de los casos con un cuadro conjunto de meningitis y sepsis/bacteriemia.

Los días de hospitalización y estancia en UCI se documentaron en 30 casos del total de 36 registrados durante los años del estudio. La media de días de hospitalización fue de 11,8 (DS 3,0). Precisaron ingreso en UCI un $50 \%$ de todos los hospitalizados por este microorganismo, con una estancia media de 3,6 (DS 0,8) de todos los casos en los que se conocía este dato (figura 2). No hubo diferencias estadísticamente significativas según el sexo en el número de días de hospitalización. No se incluye en esta valoración los niños/as fallecidos. La duración de la hospitalización según las manifestaciones clínicas queda reflejada en la tabla 4.

Evolucionaron a la curación 33 niños ( $92 \%$ de los casos), un caso presentó secue-

Tabla 3

Manifestaciones clínicas de enfermedad invasora por $\boldsymbol{H}$. influenzae (número de casos y porcentaje)

\begin{tabular}{|lcccccc|}
\hline Manifestaciones clinicas & 1996 & 1997 & 1998 & 1999 & 2000 & Total \\
\hline Meningitis & 17 & 3 & 2 & & 2 & $\mathbf{2 4}(\mathbf{6 7 \%})$ \\
Sepsis/Bacteriemia & 4 & 4 & & $\mathbf{9}(\mathbf{2 5 \%})$ \\
Neumonía & 3 & & $\mathbf{2}$ & $\mathbf{1}$ & $\mathbf{2}(\mathbf{8 \%})$ \\
\hline Total & $\mathbf{2 4}$ & $\mathbf{7}$ & & $\mathbf{3 6}(\mathbf{1 0 0 \%})$ \\
\hline
\end{tabular}




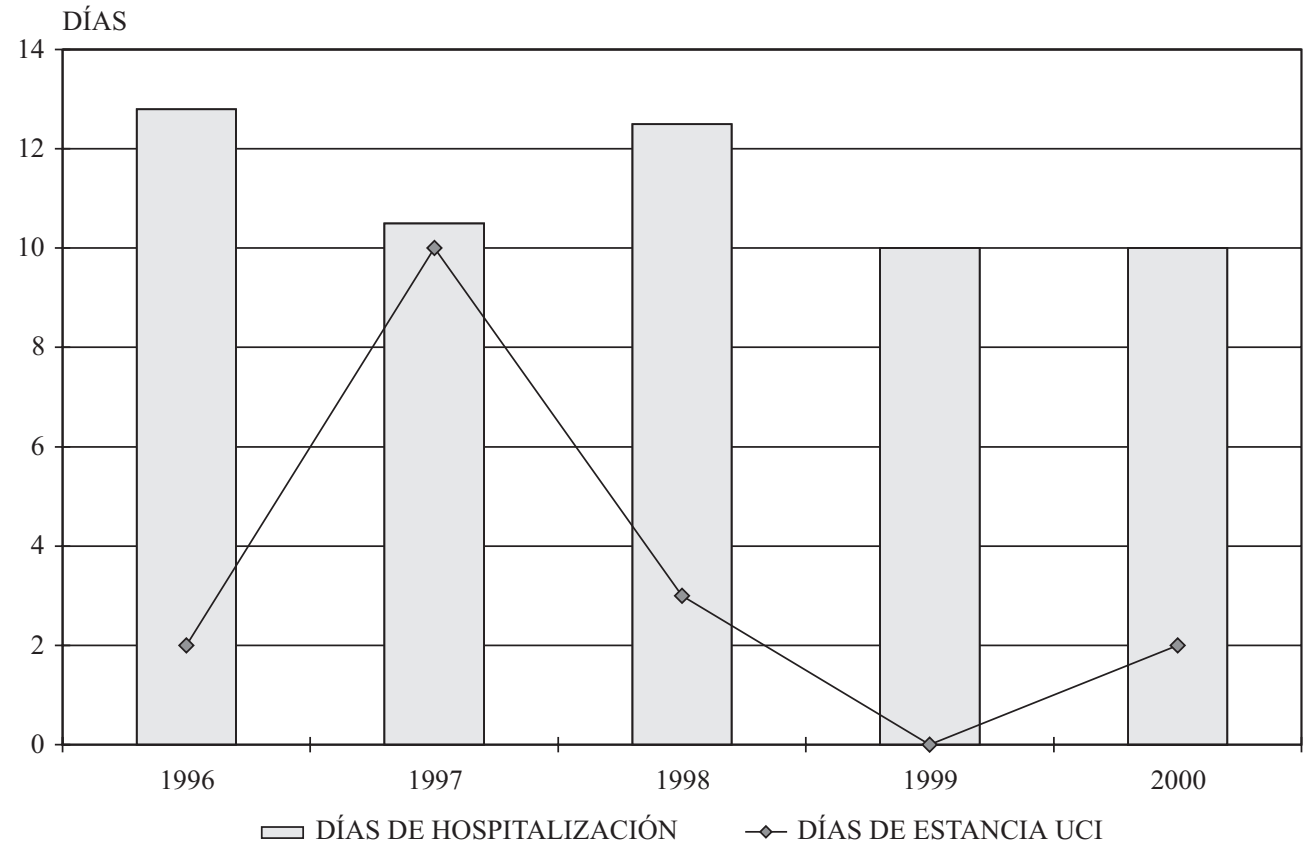

Tabla 4

Días de hospitalización según las manifestaciones clínicas que presentaron (media y desviación típica)

\begin{tabular}{|lc|}
\hline Manifestaciones clínicas & Días de hospitalización \\
\hline Meningitis & $12,7 \pm 2,4$ \\
Sepsis/Bacteriemia & $5,7 \pm 3,1$ \\
Neumonía & $8,5 \pm 4,2$ \\
\hline
\end{tabular}

las (niño de 1 año y 2 meses no vacunado, ingresado en 1996 con clínica de meningitis y que presentó alteraciones en TAC) y 2 casos evolucionaron hacia la muerte por shock séptico, ocurridos ambos en el año 1996, lo que representa una letalidad del 5,6\% para los 5 años del estudio. Estos casos corresponden a una niña de 1 año y 5 meses de edad con manifestación clínica de sepsis/bacteriemia, con antecedentes de diabetes mellitus y malnutrición y en la que no consta que estuviera vacunada frente a este microorganismo; y un niño de 9 meses de edad no vacunado que presentaba clínica de meningitis y sepsis.

El número de casos de enfermedad invasora por Hib que habían recibido vacuna conjugada frente a este microorganismo puede verse en la tabla 5. Ninguno de los niños vacunados frente al Hib y que desarrollaron enfermedad invasora por este germen falleció.

En dos de los casos (niño de 3 años/5 meses y niño de 22 meses) se identificó, en análisis posteriores, que el $H$. influenzae causante del cuadro era «no tipable» y por tanto no prevenible por vacunación actualmente (tabla $5^{*}$ y $5^{* *}$ ). Hasta la fecha desconocemos las razones del comportamiento del tercer caso (tabla $5^{* * * *}$ ).

La efectividad vacunal sobre la morbimortalidad del $H$. influenzae en la población menor de 15 años se calculó según la fórmula de Orestein (1985). En el primer año tras 
Tabla 5

Número de casos y de fallecidos de enfermedad invasora por $\boldsymbol{H}$. influenzae en función del estado de vacunación frente a este microorganismo

\begin{tabular}{|ccccccc|}
\hline \multirow{2}{*}{ Año } & \multicolumn{2}{c}{ Vacunados } & \multicolumn{2}{c|}{ No vacunados } & \multicolumn{2}{c|}{ No consta } \\
\cline { 2 - 6 } & $N{ }^{\circ}{ }^{\circ}$ casos & Fallecidos & $N^{\circ}{ }^{\circ}$ casos & Fallecidos & No consta & Fallecidos \\
\hline 1996 & $2 *$ & 0 & 16 & 1 & 6 & 1 \\
1997 & $2 * *$ & 0 & 3 & 0 & 2 & 0 \\
1998 & 1 & 0 & 1 & 0 & 0 & 0 \\
1999 & 0 & 0 & 0 & 0 & 1 & 0 \\
2000 & $1 * * *$ & 0 & 0 & 0 & 1 & 0 \\
\hline
\end{tabular}

Los asteriscos $(*)$ corresponden a casos identificados en análisis posteriores como H. influenzae no tipable.

* Corresponde a un niño de 3 años y 5 meses de edad con manifestación clínica de meningitis que había recibido una única dosis 12 meses antes del ingreso. ** Corresponde a un niño de 22 meses de edad con clínica de sepsis que había recibido 4 dosis a los 2, 4, 6, y 18 meses de edad.

*** Corresponde a un niño de 2 años y 11 meses de edad con clínica de meningitis que había recibido 4 dosis a los 2, 4, 6 y 18 meses de edad.

la inclusión de la vacuna conjugada en el calendario de vacunaciones (1998) se registraron 2 casos ( 1 en vacunado y 1 en no vacunado); la efectividad de la vacuna para el conjunto de la población en este grupo de edad fue del 75\%. En el año 1999 no se registró ningún caso entre los vacunados, la efectividad de la vacuna en este año fue del $100 \%$. En el año 2000, no nos es posible calcular la efectividad al no haberse presentado ningún caso en no vacunados.

\section{DISCUSIÓN}

Este estudio nos ha permitido obtener, simultáneamente con la valoración de la incidencia, las características clínicas más notables y la evolución epidemiológica de la enfermedad bacteriana invasora por $H$. influenzae en la población infantil de la Comunidad Valenciana entre los años 1996 y 2000. Además, hemos podido identificar dentro de los distintos grupos de edad, a aquellos niños/as que estaban en mayor riesgo de adquirir la infección y así acentuar y adecuar a ellos/as las intervenciones preventivas.

El periodo estudiado que comprende el año 1997 de la campaña de vacunación (anti-Haemophilus influenzae tipo b en menores de 5 años) así como el año anterior y los tres años posteriores a esta actuación nos ofrece una referencia objetiva de la evolu- ción epidemiológica de la enfermedad bacteriana por este microorganismo, permitiéndonos, por último, disponer de datos de la efectividad de la intervención sobre la morbilidad y mortalidad por este germen durante los años 1998, 1999 y 2000.

Se han recogido todos los casos registrados en los Servicios de Pediatría de los hospitales públicos de la Comunidad Valenciana. Podemos suponer que los casos de pacientes con enfermedad invasora por este microorganismo no incluidos en el estudio por haber recibido asistencia extrahospitalaria o por ingreso en hospitales privados deben de ser muy pocos, basándonos en que el Sistema Nacional de Salud ofrece una asistencia de calidad, universal, pública y sin coste para el paciente; en la gravedad de estos procesos; en la obligatoriedad de la declaración de enfermedad de los casos por Haemophilus influenzae tipo b y en el escaso número de camas pediátricas privadas, especialmente de cuidados intensivos.

La epidemiología de la enfermedad invasora bacteriana en la infancia se ve fuertemente influida por la administración de vacunas eficaces en la población. La evolución de la incidencia ha variado a lo largo de los 5 años del estudio. La máxima incidencia (tasa de 3,56) se observa en el año 1996. Tras la campaña de vacunación masiva frente a Hae- 
mophilus influenzae tipo b en 1997 y la posterior incorporación de la vacuna conjugada anti-Hib al Calendario de Vacunación Sistemática de la Comunidad Valenciana, se produjo una disminución drástica de casos (tasa de 0,30) en el año 1998, situación que se mantiene en estos últimos años y que coincide con lo observado en otras Comunidades Autónomas $^{25,26}$ y en otros países ${ }^{15,16, \text { y 27-30 }}$.

La enfermedad invasora bacteriana por $H$. influenzae puede darse a cualquier edad, sin embargo las mayores tasas de ataque se dan en menores de 5 años, especialmente en los primeros 18 meses de vida, como se com-

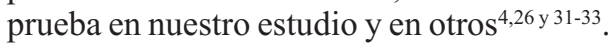

Al igual que en otros estudios, también se ha relacionado el desarrollo de enfermedad invasora por $H$. influenzae con otros antecedentes médicos de problemas de salud, fundamentalmente los déficits inmunitarios y las enfermedades crónicas de base, como sucedió en el $11,1 \%$ de los pacientes con enfermedad invasora por este microorganismo. Todos estos casos se dieron en menores de 5 años, lo que concuerda con otros trabajos donde el 95\% de las infecciones sistémicas por Hib, se producen en niños menores de 5 años ${ }^{26,33,34}$.

La presentación clínica más frecuente fue la meningitis $(67 \%)$, seguida de la sepsis $(25 \%)$ y la neumonía $(8 \%)$. El estudio de la incidencia de enfermedad invasora por Haemophilus influenzae en España ${ }^{32}$ también constató la meningitis como la presentación clínica más frecuente ( $64 \%$ de los casos) y en lo mismo concuerdan otros autore ${ }^{15,25,34}$.

La estancia media hospitalaria de 11,8 días es similar a la descrita en otros estur $\operatorname{dios}^{19,26}$. Un $50 \%$ de los hospitalizados precisaron ingreso en UCI, con una estancia media de 3,6 días.

Al alta presentaban secuelas un $2,8 \%$ del total de los niños/as a expensas de un cuadro clínico de meningitis, las secuelas documentadas se dan en menores de 2 años, lo que coincide con otros estudios. El número de se- cuelas que hemos encontrado fue inferior al esperado según otras publicaciones ${ }^{31,32}$, hecho atribuible a la disminución de la incidencia de esta infección en los últimos años.

Se ha observado una letalidad del 5,6\%, destacándose que los casos de fallecimiento se producen en niños menores de 2 años y son anteriores a la aplicación rutinaria de la vacuna conjugada anti Hib en la población infantil. Esta cifra es menor a la encontrada por Otero ${ }^{31}$ que documentó una mortalidad global del 6,2\%, mayor a la del estudio de Pachón et al de $1998^{32}$, que halló una tasa de letalidad del $4,7 \%$ y concuerda con otros estudios más recientes ${ }^{26}$.

Es difícil poder valorar a partir de nuestros datos la efectividad clínica de la vacuna frente a $H$. influenzae tipo b durante los dos primeros años del estudio, ya que en muchas ocasiones no se recoge en la historia clínica el número y fecha exacta de la administración de las distintas dosis y por otra parte desconocemos con exactitud la cobertura vacunal de los años 1996 y 1997. Debido a que los pediatras en ocasiones venían recomendándola a los niños desde su comercialización en España, en 1995, un número desconocido de niños/as ya estaban inmunizados frente a este microorganismo al comienzo de la campaña de vacunación de $1997^{18,23}$. Las coberturas vacunales de $3 .^{\mathrm{a}}$ dosis en el $1^{\mathrm{er}}$ año de vida frente al Hib alcanzadas en 1998, 1999 y 2000 fueron $80 \%$, 92\% y 93\% respectivamente. La relación de la tasa de ataque de enfermedad invasora por $H$. influenzae en niños vacunados y no vacunados, nos ha permitido calcular la efectividad clínica de la vacuna conjugada durante los últimos años. La efectividad encontrada fue del 75\% en 1998 y del $100 \%$ en 1999. En el año 2000 (donde aparece un caso sin confirmación de tipo) la efectividad vacunal no es valorable, al no registrarse ningún caso de enfermedad invasora por Hib en niños no vacunados. La drástica reducción de casos de enfermedad invasora por $H$. Influenzae, llegando casi a su eliminación, es atribuible a la alta eficacia de la vacuna, lo que observan también otros au- 
tores ${ }^{15,16,35}$. La enfermedad no sólo disminuye drásticamente en el grupo de vacunados sino también en los niños que no han recibido ninguna dosis de vacuna, al eliminar el estado de portador sano de Hib en nasofaringe, provocando una inmunidad de grupo ${ }^{36}$.

Sin embargo, es indicación de fallo vacunal la detección de 1 caso en 1996 y 1 caso en 1997 de enfermedad invasora por $H$. influenzae en niños que habían recibido un número adecuado de dosis para su edad $\mathrm{y}$ que permitió considerarles correctamente inmunizados; los estudios microbiológicos posteriores revelaron que correspondían a $H$. influenzae tipo no b. Desconocemos hasta el momento las razones del comportamiento del tercer caso detectado en el año 2000. La aparición de casos de H. influenzae tipo no b, observado también por otros autores $^{16,26}$ justifica la necesidad de continuar vigilando la enfermedad invasora causada por este microorganismo.

Dado los excelentes resultados obtenidos con la incorporación al Calendario de Vacunaciones Sistemáticas de la Comunidad Valenciana de la vacuna conjugada contra $H$. influenzae en cuanto a morbimortalidad, sobre todo, en niños menores de 2 años y en niños con alguna enfermedad predisponente y/o antecedente de problema de salud, creemos conveniente seguir asegurando unas altas coberturas de esta vacuna en la población infantil.

\section{AGRADECIMIENTOS}

La realización de este estudio ha sido posible gracias a la dedicación y el esfuerzo realizado por el personal facultativo de los Servicios de Pediatría de los hospitales públicos de la Comunidad Valenciana y al apoyo prestado por la Dirección General de Salud Pública.

\section{BIBLIOGRAFÍA}

1. Shapiro ED, Ward JI. The Epidemiology and Prevention of Disease Caused by Haemophilus in- fluenzae Type b. Epidemiol Rev 1991; 13: 113-142.

2. Roca J, Campos G, Monsó G, Trujillo A, Riverola A, Suris JC et al. Meningitis en pediatría. Estudio clínico y epidemiológico de 173 casos. Enf Infec y Microbiol Clin 1992; 10: 79-88.

3. De Juan F, Campos C, Bustillo A. Infecciones invasivas por Haemophilus influenzae tipo b en la infancia. Ann Esp Pediatr 1993; 39: 111-115.

4. Bueno M, Baselga C et al (Comité de Expertos de la Asociación Española de pediatría). Meningitis bacteriana en la infancia. Situación actual en España. Epidemiología y prevención. Madrid: Gabinete de estudios Bernard Krief; 1994.

5. Stephen L, Cochi SL and Ward JI. Haemophilus influenzae Type b. In: A.S. Evans and P.S. Brachman, eds. Bacterial Infections of Humans. Epidemiology and Control. Plenum Medical Book Company; 1991. p. 277-315.

6. Moraga Llop FA. Vacunas frente a Haemophilus influenzae tipo b. En: Camping Martí M, Moraga Llop FA (eds). Prous Science Barcelona; 1997. p. 63-75.

7. Fritzell B. Efficacy and safety of a Haemophilus influenzae type b capsular polyssacharide-tetanus protein conjugate vaccine. J Pediatr 1992; 121 : 355-62.

8. Eskola J, Peltola H, Takala AK et al. Efficacy of Haemophilus influenzae type $\mathrm{b}$ polysaccharide/diphtheria toxoide conjugate vaccine in infancy. N Engl J Med 1987; 317: 717-22.

9. Decker MD, Edwards KM, Bradley R, Palmer P. Comparative trial in infants of four conjugate Haemophilus influenzae type $\mathrm{b}$ vaccines. J Pediatr 1992; 120: 184-89.

10. American Academy of Pediatrics. Committee of infections Disease. Recommended Childhood immunization schedule United State. January-Decembrer 1997. Pediatrics 1997; 99(1): 136-43.

11. Brines J, Diez J, Morant A. Introducción a la epidemiología de las enfermedades invasoras en la Comunidad Valenciana y su reflexión vacunal. Bol Soc Val Pediatr 1998; 18: 157-65.

12. Diario Oficial de la Generalitat Valenciana. Orden de 17 de octubre de 1997, de la Conselleria de Sanidad, por la que se aprueba el nuevo calendario de vacunaciones infantiles en la Comunidad Valenciana. DOGV núm 3134, 16/12/1997.

13. Committee of Infectious Disease. Haemophilus influenzae type b conjugate vaccines: Recommendations for immunization with recently and 
previously licensed vaccines. Pediatrics 1993; 92: $480-8$

14. Advisory Committee on Immunization Practices (ACIP). General recommendations on immunization. MMWR 1994; 43: 1-38.

15. Peltola H. Worldwide Haemophilus influenzae type $\mathrm{b}$ disease at the beginning of the $21^{\text {st }}$ century: Global analysis of the disease burden 25 years after the use of the polysaccharide vaccine and a decade after the advent of conjugates. Clin Microbiol Rev 2000; 13: 302-317.

16. Perdue D, Bulkow L, Gellin B, Davidson M, et al. Invasive Haemophilus influenzae disease in Alaska residents aged 10 years and older before and after infant vaccination programs. Jama 2000; 23 . 3089-3094.

17. Diario Oficial de la Generalitat Valenciana Orden de 4 de marzo de 1997, de la Conselleria de Sanidad, por la que se desarrolla el Sistema Básico de la Red Valenciana de Vigilancia en Salud Pública. DOGV núm 2.983, 2/5/1997.

18. Redondo MJ, Momparler P, Fullana A, Lluch J, Bueno F. Estimación de la incidencia de enfermedad invasora por Haemophilus influenzae en la Comunidad Valenciana a través del sistema de información hospitalaria C.M.D.B. XI Jornadas de Salud pública y Administración sanitaria. VII Simposium de Salud Materno-Infantil. Granada 17-19 noviembre 1996. Libro del Congreso. p. 103-4.

19. García V, Moreno C. Estudio de la incidencia de enfermedad invasiva por Haemophilus influenzae en niños menores de 5 años en Navarra. 1991-1994. Bol Salud Pública de Navarra 1996; 17: 7-11.

20. Incidencia de meningitis bacteriana y enfermedades invasivas por Haemophilus influenzae. Bol Epidemiol Comunidad de Madrid 1994; 4: 3-27.

21. Resum de les malalties de declaració obligatoria durant l'any 1994. Bull Epidemiol Catalunya 1995; XVI. p. 95-112.

22. Estudio sobre la incidencia de infección por Haemophilus influenzae tipo b realizados en España: Datos facilitados por las respectivas Comunidades Autónomas (Informe interno).. Madrid: Centro Nacional de Epidemiología; 1995.

23. Generalitat Valenciana. Conselleria de Sanitat Plan de prevención integral de la meningitis en la Comunidad Valenciana. Valencia: Generalitat Valenciana; 1999.

24. Orestein W, Bernier R, Sondero T, Hinman A, Mark J, Bart K et al. Field evaluation of vaccine efficacy. Bull World Health Organ 1985; 63: 1055-68.
25. Bachiller P, Eiros JM, Blanco A. Clinical manifestations, diagnosis and treatment of Haemophilus influenzae infection. An Med Interna 2000; 17 (4): 204-212.

26. Simarro E, Ruiz J, Gómez J, Ortega MG, Vicente C, Martínez L et al. Haemophilus influenzae infections in children less than 5 years of age in the community of Murcia during the 1992-1999 period. Enferm Infecc Microbiol Clin 2000; 18 (7): 325-328.

27. Lee A, Crutcher J. Oklahoma notes decline in Haemophilus influenzae: invasive Haemophilus influenzae disease among children aged $<5$ years, Oklahoma, 1990-1997. J Okla State Med Assoc 1999; 92(6): 276-277.

28. Lolekha S, Cooksley G, Chan V, et al. A review of Hib epidemiology in Asia. Southeast Asian Trop Med Public Health 2000; 31 (4): 650-657.

29. Rosenstein N, Perkins B. Update on Haemophilus influenzae serotype $\mathrm{b}$ and meningococcal vaccines. Pediatr Clin North Am 2000; 47 (2): 337-352.

30. Singleton R, Bulkow L, Levine O et al. Experience with the prevention of invasive Haemophilus influenzae type $\mathrm{b}$ disease by vaccination in Alas$\mathrm{ka}$ : the impact of persistent oropharyngeal carriage. J Pediatr 2000; 137: 313-320.

31. Otero MC. Importancia del Haemophilus influenzae en la patología pediátrica [Tesis doctoral]. Valencia: Universidad de Valencia; 1993.

32. Pachón I, Muñoz A, Tormo A, Amela C, Martín $\mathrm{P}$, Villota $\mathrm{J}$ et al. Estudio de la incidencia de enfermedad invasora por Haemophilus influenzae en España. Bol Epidemiol Sem 1998; 6: 49-52.

33. Díez Domingo J, Pereiró Berenguer I, Ferrer Salvá $\mathrm{A}$, et al. Cobertura de las vacunas administradas sistemáticamente y de la vacuna frente al $H$ influenzae tipo b previa a su incorporación al calendario vacunal en la C.V. An Esp Pediatr 1998; 49: $568-570$.

34. Otero MC, Nieto A. Infección invasora por Haemophilus influenzae tipo b. ¿Curar o prevenir? Rev Esp Quimioterap 1995; 8(Pt 1): 18-20.

35. Garpenholt O, Hugosson S, Fredlund H, Giesecke J, Olcen P. Invasive disease due to Haemophilus influenzae type $\mathrm{b}$ during the first six years of general vaccination of Swedish children. Acta Pediatric 2000; 89(4): 471-474.

36. Eskola j, Käyhty H. Ten years experience with Haemophilus influenzae type $\mathrm{b}$ (Hib) conjugate vaccine in Finland. Rev Med Microbiol 1996; 7 : 231-41 